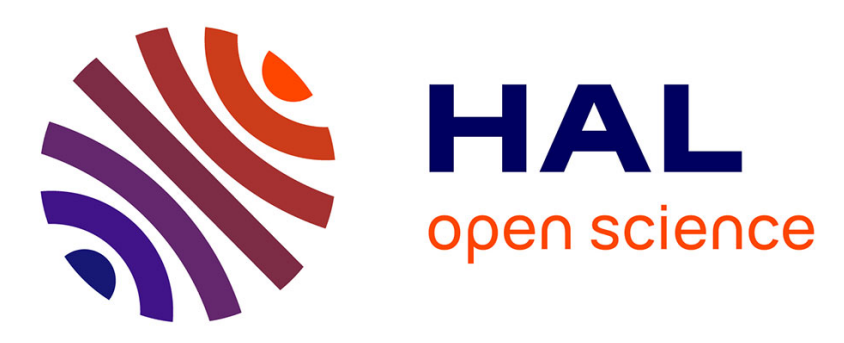

\title{
Genetic and Genomic Mechanisms of Primary Aldosteronism
}

Fabio L Fernandes-Rosa, Sheerazed Boulkroun, Maria-Christina Zennaro

\section{To cite this version:}

Fabio L Fernandes-Rosa, Sheerazed Boulkroun, Maria-Christina Zennaro. Genetic and Genomic Mechanisms of Primary Aldosteronism. Trends in Molecular Medicine, 2020, 26 (9), pp.819-832. 10.1016/j.molmed.2020.05.005 . inserm-03554636

\section{HAL Id: inserm-03554636 https://www.hal.inserm.fr/inserm-03554636}

Submitted on 3 Feb 2022

HAL is a multi-disciplinary open access archive for the deposit and dissemination of scientific research documents, whether they are published or not. The documents may come from teaching and research institutions in France or abroad, or from public or private research centers.
L'archive ouverte pluridisciplinaire HAL, est destinée au dépôt et à la diffusion de documents scientifiques de niveau recherche, publiés ou non, émanant des établissements d'enseignement et de recherche français ou étrangers, des laboratoires publics ou privés. 
Genetic and genomic mechanisms of primary aldosteronism

Fabio L. Fernandes-Rosa ${ }^{1}$, Sheerazed Boulkroun ${ }^{1}$, Mariani-Christina Zennaro ${ }^{1,2}$

\author{
Affiliations: \\ ${ }^{1}$ Inserm, PARCC, Université de Paris, F-75015 Paris, France \\ ${ }^{2}$ Assistance Publique-Hôpitaux de Paris, Hôpital Européen Georges Pompidou, Service \\ de Génétique, Paris, France
}

Address correspondence to:

Maria-Christina Zennaro, MD, PhD

INSERM, U970

Paris Cardiovascular Research Center - PARCC

56, rue Leblanc,

75015 Paris - France

Tel : +33(0)1 53988042

Fax : + $33(0) 153987952$

e-mail: maria-christina.zennaro@inserm.fr

\title{
Disclosures
}

The authors have nothing to disclose. 


\begin{abstract}
Aldosterone producing adenomas (APA) and bilateral adrenal hyperplasia are the main cause of primary aldosteronism (PA), the most frequent form of secondary hypertension. Mutations in ion channels and ATPases have been identified in APA and inherited forms of PA, highlighting the central role of calcium signalling in PA development. Different somatic mutations are also found in aldosterone producing cell clusters in adrenals from normal subjects and from patients with unilateral and bilateral PA, suggesting additional pathogenic mechanisms. Recent mouse models have also contributed to a better understanding of PA. Application of genetic screening in familial PA, development of surrogate biomarkers for somatic mutations in APA and use of targeted treatment directed at mutated proteins may allow improved management of patients.
\end{abstract}


Primary aldosteronism (PA) is the most frequent form of secondary arterial hypertension with a prevalence of $5 \%$ of hypertensive patients in primary care and until $10 \%$ of hypertensive subjects in reference centers $[1,2]$. PA is due to autonomous aldosterone production by the adrenal gland, the two major causes being the presence of an aldosterone producing adenoma (APA) or bilateral adrenal hyperplasia (BAH). In addition to sporadic forms, four different familial forms were described, accounting for approximately 5\% of PA cases. PA patients exhibit hypertension associated in many cases with hypokalemia. The biochemical diagnosis is based on high levels of plasma aldosterone and low plasma renin, with an increased aldosterone to renin ratio (ARR). The confirmation of PA diagnosis is performed by one of different tests, including oral salt loading or captopril challenge tests, followed by adrenal image and adrenal vein sampling (AVS) to subtype PA as unilateral or bilateral forms [3]. PA subtyping guides the optimal treatment, unilateral adrenalectomy for lateralized APA or long-life drug therapy with mineralocorticoid receptor antagonists in the presence of bilateral forms. Due to deleterious effects of aldosterone on blood vessels and heart, patients with PA have an increased cardiovascular risk when compared with age, sex and blood pressure matched patients with primary hypertension $[4,5]$.

Aldosterone is synthetized from cholesterol in the cells of the zona glomerulosa (ZG) of the adrenal cortex by a cascade of enzymatic reactions. The ZG specifically expresses the enzyme aldosterone synthase (encoded by $C Y P 11 B 2$ ), responsible for the three final enzymatic reactions leading to aldosterone biosynthesis [6]. Aldosterone biosynthesis is tightly regulated to maintain salt and water homeostasis, the two most important factors being angiotensin II (AngII) and extracellular potassium concentration $\left(\mathrm{K}^{+}\right)$(Box 1). In basal conditions $\mathrm{ZG}$ cells are maintained in a hyperpolarized state due to the presence of a large number of potassium channels [7] and the $\mathrm{Na}^{+} / \mathrm{K}^{+}$-ATPase. ZG cell membrane depolarization is induced by AngII or increase in plasma $\mathrm{K}^{+}$concentration, causing the opening of voltage-gated $\mathrm{Ca}^{2+}$ channels and the increase of the intracellular $\mathrm{Ca}^{2+}$ concentration. AngII also stimulates $\mathrm{Ca}^{2+}$ release from the endoplasmic reticulum by binding the angiotensin II Type 1 receptor (AT1R) and stimulating the inositol triphosphate pathway. The increase in cytosolic $\mathrm{Ca}^{2+}$ activates calcium signaling (via calmodulin and calcium/calmodulin-dependent protein kinases), the main trigger for CYP11B2 expression and aldosterone production [8, 9]. Other regulators of aldosterone biosynthesis include ACTH, serotonin, interleukine 6, epidermal growth factor and leptin [10$13]$. 
In the last decade, following the discovery of $K C N J 5$ mutations associated with PA, different studies performing whole exome sequencing identified mutations in genes coding for ion channels and ATPases as responsible for APA and familial PA and highlighted the central role of calcium signaling on PA pathogenesis. These studies allowed the identification of somatic mutations in more than $85 \%$ of APA and completely changed the classification of familial forms (Box 2. Clinician's Corner).

\section{Genetics of primary aldosteronism}

The first genetic defect associated with PA was described almost 30 years ago. A chimeric gene, where the regulatory regions of $C Y P 11 B 1$ are juxtaposed with the coding sequence of CYP11B2, was identified as responsible of familial hyperaldosteronism Type 1 (FH-I) $[14,15]$ (Table 1). FH-I is an autosomal dominant disease presenting as early-onset hypertension associated with biochemical abnormalities of PA and increased production of 18hydroxycortisol and 18-oxocortisol, which improves with glucocorticoid treatment [16, 17]. The chimeric $C Y P 11 B 1 / C Y P 11 B 2$ gene leads to ectopic expression of $C Y P 11 B 2$ throughout the adrenal cortex, with aldosterone being produced not only in cells from ZG, and with an inappropriate $\mathrm{ACTH}$-dependent regulation of aldosterone biosynthesis following the circadian rhythm of cortisol (Figure 1) [18, 19].

During the last decade, whole exome sequencing (WES) performed on DNA from APA has allowed the identification of somatic mutations in genes coding for ion channels (KCNJ5 encoding for the potassium channel GIRK4, CACNA1D coding for the $\alpha 1$ subunit of the L-type $\mathrm{Ca}^{2+}$ channel Cav1.3) and ATPases (ATP1A1, coding for the $\alpha 1$ subunit of the $\mathrm{Na}^{+}, \mathrm{K}^{+}$-ATPase, and $A T P 2 B 3$, coding for the plasma membrane calcium-transporting ATPase 3 (PMCA3) [2023]. The frequency of somatic mutations was reported to be approximately $50 \%$ in studies performing Sanger sequencing of hot spot regions of APA-driver genes on DNA extracted from frozen pieces of tumor [24, 25]. The recent development of aldosterone synthase (CYP11B2) immunohistochemistry (IHC)-guided next-generation sequencing (NGS) allowed the identification of mutations in APA-driver genes in $88 \%$ to $93 \%$ of APA [26-28].

In parallel, the discovery of germline mutations in genes coding for the same ion channels identified in APA and also in CACNA1H (coding for the $\alpha 1$ subunit of the T-type Ca2+ channel Cav3.2) and CLCN2 (coding for the chloride channel ClC-2) have complexified the 
classification of familial forms of PA [20, 22, 29-32], with four different forms now classified in FH-I to FH-IV, based on the underlying genetic defect.

All the different somatic and germline mutations increase CYP11B2 expression and aldosterone biosynthesis by affecting cell membrane potential and/or intracellular ionic homeostasis, resulting in increased intracellular calcium concentration and activation of calcium signaling (Figure 1).

\section{KCNJ5 mutations}

Somatic and germline $K C N J 5$ mutations were identified in APA and in patients with a familial form of PA (FH-III) [20]. The most prevalent KCNJ5 mutations (p. Gly151Arg, p. Thr158Ala, p. Leu168Arg) are localized near or within the selectivity filter of the channel. These mutations cause loss of $\mathrm{K}^{+}$selectivity and increase sodium permeability, resulting in cell membrane depolarization followed by opening of voltage-gated $\mathrm{Ca}^{2+}$ channels and stimulation of $\mathrm{Ca}^{2+}$ signaling [20, 33, 34] (Figure 1). A small number of $K C N J 5$ mutations are localized in regions far from the selectivity filter and could be responsible for decreased abundance of the channel at the plasma membrane [35]. While the impact of GIRK4 downregulation on aldosterone production was not observed in cell models [33], a recent study suggested that cell proliferation and apoptosis may be regulated by the level of GIRK4 expression [36].

Recurrent somatic KCNJ5 mutations are the most frequent genetic abnormality observed in APA, being identified in approximately $40 \%$ of adenomas [24, 37, 38]. Ethnic differences were observed among cohorts, with a higher prevalence of somatic KCNJ5 mutations in patients from East Asian cohorts [35, 39-41]. Different studies have shown that KCNJ5 mutations are more frequent in female and younger patients [24-28, 35, 38, 42, 43], and may also be associated with higher aldosterone levels and larger tumor size in some studies $[37,38]$ but not in others [28]. KCNJ5 mutations were also suggested to be a marker of better surgical outcome after adrenalectomy [44]. Compared with other mutational groups, APA carrying KCNJ5 mutations present lower percentage of cells expressing CYP11B2 and higher percentage of cells expressing CYP11B1 [28]. A recent study, however, reported no difference in overall aldosterone synthase expression as a function of mutational status [45]. In addition, KCNJ5mutated APA exhibit decreased GIRK4 expression in comparison with the adjacent ZG [28, $36,46,47]$.

Germline KCNJ5 mutations are also responsible for FH-III [20] (Table 1). FH-III was first described in 2008 as severe early-onset hypertension associated with hypokalemia, high urinary concentrations of hybrid steroids 18-oxocortisol and 18-hydroxycortisol, and massive bilateral 
adrenal hyperplasia [48]. Germline KCNJ5 mutations were found in different FH-III families presenting variability in the severity of hyperaldosteronism and in the subtype of PA, sometimes within the same family [49-53].

In vitro studies revealed that mutated GIRK4 may be blocked by high therapeutic doses of the calcium channel blocker verapamil, or by macrolides, decreasing CYP11B2 expression and aldosterone biosynthesis [54-56]. Clinical studies are currently ongoing, in hypertensive patients with PA, to evaluate the potential clinical use of macrolides on PA subtyping or the identification of somatic KCNJ5 mutations before surgery [57].

\section{CACNA1D and CACNA1H mutations}

CACNA1D mutations affect different properties of Cav1.3 channel, delaying the voltagedependent inactivation or inducing opening of the channel at less depolarized potentials. This results in increased $\mathrm{Ca}^{2+}$ influx leading to the activation of $\mathrm{Ca}^{2+}$ signaling and aldosterone overproduction (Figure 1) [21, 22]. Using CYP11B2 IHC-guided NGS, the prevalence of somatic CACNA1D mutations was $21 \%$ in APA from white American patients, $37 \%$ in a French cohort, and as high as $42 \%$ in APA from African-American patients, being the most frequent genetic abnormality in this population [26-28]. While CACNA1D mutations were associated with smaller tumors in studies using Sanger sequencing to genotype APA [24], this association was not described in a recent study using CYP11B2 IHC-guided NGS [28]. In addition to somatic mutations, de novo germline mutations in CACNAID were identified in two children exhibiting a severe form of hyperaldosteronism in the first year of life associated with a complex neurologic disorder (Primary Aldosteronism, Seizures and Neurologic Abnormalities (PASNA) [22] (Table 1).

Germline CACNA1H mutations are responsible for FH-IV. Mutations modify the Cav3.2 channel properties inducing a gain of function, resulting in increased $\mathrm{Ca}^{2+}$ influx followed by activation of calcium signaling and overproduction of aldosterone (Figure 1) [29, 30]. The recurrent germline $C A C N A 1 H$ mutation p.Met1549Val was described in five children with onset of PA before age 10 years [29]. Micronodular adrenal hyperplasia was observed in the histological analysis of one case [29]. A de novo germline CACNA1H mutation also affecting codon 1549 (p.Met1549Ile) was described in one children with PA associated with multiplex developmental disorder [30]. Germline CACNA1H mutations were also identified in adult patients with a familial inheritance of PA and in one patient with APA, suggesting that CACNAIH mutations may predispose to PA with different phenotypic presentation [30]. Recently, somatic CACNA1H mutations were also observed in a rare subset of APA [58]. 
ATP1A1 mutations were identified in $8 \%$ to $17 \%$ of APA by CYP11B2 IHC-guided NGS [26-28]. The $\alpha$ subunit of the $\mathrm{Na}^{+}, \mathrm{K}^{+}$-ATPase, encoded by ATP1A1, is composed of 10 transmembrane domains (M1 to M10) and intracellular N- and C- termini. Somatic ATP1A1 mutations are localized in M1, M4 and M9 domains [21, 23, 25, 40, 59]. While mutations in the M1 and M4 domains affect the $\mathrm{K}^{+}$binding pocket decreasing the pump affinity for $\mathrm{K}^{+}$, mutations in the M9 domain affect a supposed sodium-specific site of the pump, all mutations decreasing the pump activity $[60,61]$. The reduced affinity for $\mathrm{K}^{+}$and the loss of pump activity result in cell membrane depolarization and cell acidification, without overt increase of intracellular $\mathrm{Ca}^{2+}$ (Figure 1) [60]. However, the role of cytosolic acidification on autonomous aldosterone production is not established.

ATP2B3 mutations were identified in $1.7 \%$ to $10 \%$ of APA, with higher prevalence in studies using CYP11B2 IHC-guided NGS [24-28, 43]. PMCA3, encoded by ATP2B3 is composed of 10 transmembrane domains (M1 to M10) and intracellular N- and C-termini. All APA-driver ATP2B3 mutations are in frame deletions affecting a "PEGL" motif in the M4 domain, a region involved in calcium binding and ion gating [23-25, 43, 62]. Mutations in $A T P 2 B 3$ decrease pump activity, impairing calcium export and resulting in increased cytosolic calcium concentration and activation of calcium signaling (Figure 1). A second mechanism for autonomous aldosterone biosynthesis was suggested; $A T P 2 B 3$ mutations may induce a sodium leak resulting in cell membrane depolarization and opening of voltage-dependent calcium channels, but may also induce a calcium leak through mutated pumps [63].

\section{CLCN2 mutations}

Germline CLCN2 mutations are responsible for FH-II (Table 1). FH-II was used to group all familial forms of PA without an established genetic cause, with a diagnosis based on the presence of two or more affected family members [64, 65]. Whole-exome sequencing performed in a large Australian FH-II family identified a germline mutation in CLCN2 segregating with the disease in 8 subjects [32]. Three other PA pedigrees carried the same mutation and, additionally, four different germline CLCN2 mutations were identified in unrelated patients with early-onset PA [32]. A de novo germline CLCN2 mutation was identified, concomitantly, in a 9 years-old PA patient exhibiting severe hypertension and profound hypokalemia [31]. This mutation changes the properties of ClC-2 leading to increased chloride conductance at resting potentials and chloride efflux followed by cell membrane 
depolarization, activation of calcium signaling and stimulation of $C Y P 11 B 2$ expression and autonomous aldosterone production (Figure 1) [31]. In addition to germline mutations and FHII, a somatic CLCN2 mutation was reported in one APA [66].

\section{CTNNB1, PRKACA and ARMC5 mutations}

Somatic mutations in CTNNB1, coding for $\beta$-catenin, were identified in $2.1 \%$ to $5.1 \%$ of APA [43, 67]. CTNNB1 mutations are localized in exon 3 and were previously described in cortisol-producing adenomas and adrenocortical cancer. CTNNB1 mutations are more prevalent in women and an association with PA during pregnancy due to aberrant expression of gonadal receptors in APA was suggested [68]. However, it has been shown that the regulation of aldosterone production by gonadal hormones may also occur in APA without somatic CTNNB1 mutations [69]. Further studies are necessary to establish the role of CTNNB1 mutations on APA development.

Somatic PRKACA (encoding the cAMP-dependent protein kinase catalytic subunit alpha) mutations were identified in two patients with APA, one presenting aldosterone and cortisol cosecretion [70]. PRKACA mutations were also described in cortisol producing adenomas and their roles in autonomous aldosterone production and APA development are unknown.

Finally, germline ARMC5 (armadillo repeat containing 5) variants, predicted to be damaging, were observed in African-American patients with PA [71]. Germline ARMC5 variants were also observed in a European cohort of patients with PA, although they were not predicted to be damaging using bioinformatics tools [72]. ARMC5 mutations were previously identified in primary bilateral macronodular adrenal hyperplasia and associated with hypercortisolism [73]. The link between ARMC5 mutations and PA remains to be further determined.

\section{Animal models of primary aldosteronism}

Genetic studies have greatly contributed to a better understanding of the mechanisms responsible for PA development, highlighting the role of ion channels and pumps, but also of the Wnt/ $\beta$-catenin pathway (Table 2). Remarkably, potassium channels and the $\mathrm{Wnt} / \beta$-catenin pathway had been shown previously to play crucial roles in the regulation of aldosterone biosynthesis and homeostasis of the adrenal cortex by specific mouse models.

Invalidation of $k c n k 3$ or/and $k c n k 9$, coding for the two-pore domain potassium channels Task 1 and Task3, resulted in different forms of hyperaldosteronism or low renin hypertension 
[74-78]. Simultaneous invalidation of $k c n k 3$ and $k c n k 9$ leads to ZG cell depolarization and PA resistant to salt suppression, without affecting cellular morphology and adrenal zonation [75]. ZG-specific invalidation of both potassium channels resulted in a milder phenotype consisting of a moderate increase of plasma aldosterone that was not associated with a reduction of plasma renin or increased ARR; nevertheless, it was sufficient to significantly increase blood pressure [78]. Different phenotypes were observed when Kcnk3 or kcnk9 were invalidated separately. Task3 channel deletion causes salt-sensitive hypertension with low renin [76, 77], associated with ZG cell depolarization, modification of $\mathrm{Ca}^{2+}$ signaling and alteration of the physiological regulation of aldosterone, without changes in adrenal morphology. In neonatal mice, Task3 deletion led to major adrenal dysfunction, associated with increased aldosterone, corticosterone and progesterone levels [79]. Deletion of Task1 resulted in severe hyperaldosteronism remediable by glucocorticoids associated with abnormal expression of aldosterone synthase in the zona fasciculata (ZF), despite normal morphological zonation of the adrenal cortex [74]. Whereas this phenotype was observed in female mice all along their life, it was only observed in pre-pubertal male mice, suggesting an androgen-driven upregulation of compensatory mechanisms [74]. In these mice, a subset of genes was closely associated with hyperaldosteronism, including $D k k 3$ coding for Dickkopf3, a member of the Wnt signaling pathway [80]. Interestingly, invalidation of $d k k 3$ in $k c n k 3^{-/-}$mice resulted in hyperaldosteronism also in male mice, suggesting a role for $d k k 3$ in the development of PA [80].

After the identification of $C L C N 2$ mutations in patients with FH-II, two different knock-in mouse models provided important insight regarding the role of chloride channels in PA development $[81,82]$. Deletion of eight $\mathrm{N}$-terminal residues constituting the inactivation domain of the Clc-2 chloride channel, where the p.Met22Lys, p.Gly24Asp and p.Tyr26Asn mutations are located [31, 32], resulted in hypertension and hypokalemia in mice, with high aldosterone and low renin levels, recapitulating the major features of PA [81]. Mice harboring the heterozygous p.Arg180Gln mutation, mimicking the human p.Arg172GIn mutation found in FH-II [32], present a mild form of PA with increased aldosterone associated with a mild elevation of blood pressure, but absence of morphological adrenal abnormalities [82].

Through different mechanisms, the mutations identified in APA converge upon increased calcium signaling to increase aldosterone production. The constitutive expression in mice of a gain-of-function mutant of the AII receptor type 1A (AT1R) led to the development of lowrenin hypertension and relative hyperaldosteronism [83]. More recently, specific activation of Gq signaling in adrenal cortex, using a Designer Receptors Exclusively Activated by Designers 
Drugs (DREADD) activated by clozapine N-oxide (CNO), was used to establish a mouse model of PA [84]. CNO treatment results in increase of Cyp11b2 expression and aldosterone levels, decreased plasma renin concentration and abnormal zonation of Cyp11b2, with ZF cells expressing both Cyp11b1 and Cyp11b2. In addition, high blood pressure was observed after CNO treatment in mice submitted to high salt diet.

Whereas these mouse models develop hyperaldosteronism, the presence of tumors was never observed, suggesting that modification of intracellular ion balance is not sufficient to induce both increase of aldosterone production and adenoma development. Activation of the Wnt/ $\beta$-catenin pathway, playing an important role in adrenal development, has been involved in adrenal carcinoma and adenoma [85] and was found in two-thirds of APA [86]. In mice, constitutive activation of $\beta$-catenin results in adrenal hyperplasia and dysplasia as well as increased cell proliferation. More interestingly, $\beta$-catenin activation also led to increased aldosterone production and hyperaldosteronism [87]. However, the development of tumors was observed only in rare cases with features of carcinoma rather than adenoma [87].

Recently, retinoic acid receptor $\alpha(\mathrm{RAR} \alpha)$ signaling has been identified as involved in nodule formation by transcriptome analysis performed in APA. Some years ago, proteomic analyses have suggested a role of retinoic acid receptor signaling in the early stage of adrenal development [88]. Invalidation of rara expression in mice results in structural disorganization of the adrenal cortex characterized by the loss of the radial organization of the ZF and abnormalities in vessel architecture and extracellular matrix, in both male and female mice, with no major modifications of aldosterone production [89]. Beyond the role of rara in the maintenance of the normal adrenal cortex structure and cell proliferation, dysregulation of this signaling may contribute to abnormal cell proliferation in PA [89].

\section{$A P C C$ and their role in aldosterone biosynthesis and $P A$}

Until the description of aldosterone producing cell clusters (APCC), expression of CYP11B2 and aldosterone biosynthesis was thought to occur exclusively in ZG cells under the tight control of different factors, the two majors being AngII and $\mathrm{K}^{+}$(see above). APCC have been described in 2010 [90]. They are located under the capsule and composed of ZG-like cells in the outer part and of columnar ZF-like cells in the inner part. Despite their morphological properties and the fact that Disabled-2, a marker of ZG, is expressed only in the outer part, the entire structure expresses CYP11B2 but not CYP11B1, suggesting that APCC may constitute 
intermediate structure between ZG and ZF $[86,91]$. However, transcriptome profiling of APCC revealed a greater homology to ZG cells than to ZF cells $[92,93]$. APCC are found in normal adrenals and their number and size are correlated with age, but not with gender [45]. APCC are also found in adrenal cortex adjacent to an APA and in adrenals from patients with BAH [90, 91, 94].

Genetic analysis of APCC, using CYP11B2 IHC-guided guided NGS on FFPE tissue, identified somatic mutations in APA driver genes, including CACNA1D, ATP1A1 and ATP2B3, but not $K C N J 5$, in 35\% of APCC from normal adrenals [92]. More recently, the description of APCC-to-APA translational lesions (pAATL), composed of a subcapsular APCC-like structure and a micro-APA-like structure and carrying somatic mutations suggested the possibility for APCC to constitute the starting point for the development of APA (Figure 2) [93]. Other evidences, however, support the occurrence of two independent events leading to APA development [95]. It has been shown that the adrenal cortex adjacent to an APA may exhibit ZG hyperplasia, increased nodulation and reduced vascularization [86]. Distinct CYP11B2 positive nodules may carry different mutations within the same adrenal $[28,96]$ and different APCC located in the cortex adjacent to an APA may also carry different somatic mutations, including KCNJ5 mutations [28]. Finally, a patient with lateralized PA and familial adenomatous polyposis further supports a two-hit model for APA development. The patients carried a germline APC mutation with loss of heterozygosity in adrenals (first hit), responsible for a macronodular hyperplasia. In one CYP11B2 positive nodule, a somatic KCNJ5 mutation was identified, being responsible for autonomous aldosterone production (second hit) (Figure 2) [97].

APCC appear also to play a role in the pathogenesis of bilateral forms of PA. In a study investigating adrenals from 15 patients with $\mathrm{BAH}$ who underwent adrenalectomy, the number of APCC was significantly higher compared to adrenals from normotensive controls, suggesting that APCC, through autonomous aldosterone production, may contribute to the development of PA. Interestingly, sequencing of 99 APCC from the 15 adrenals identified somatic mutations in at least one APCC in all but one case; all mutations affected the CACNA1D gene [94]. These results suggest for the first time that the presence of APCC in BAH may be, in part, responsible for PA development; however, they need to be confirmed in a larger cohort of patients.

\section{Concluding remarks}


Over the last years, CYP11B2 guided NGS has allowed the identification of somatic mutations in APA-driver genes in more than $85 \%$ of APA. In addition, the description of germline mutations in genes coding for different ion channels has allowed refining the classification of familial forms of PA and the description of de novo early-onset PA. In mechanistic terms, these mutations highlight the central role of plasma membrane potential, intracellular calcium concentrations and activation of calcium signaling in the pathogenesis of PA. However, the sequence of events leading to APA formation as well as the mechanisms responsible for bilateral PA remains unknown. Somatic mutations in APA-driver genes were also identified in APCC from normal adrenals and from adrenals with APA. While this discovery suggests a possible role of APCC in PA, the identification of different mutations among APA, APCC and micronodules within the same adrenal implies the contribution of additional mechanisms (see Outstanding Questions). Finally, the investigation of different mouse models has largely contributed to our understanding of some aspects of the development of hyperaldosteronism. However, none of these models leads to APA development, suggesting that there might be the requirement for additional events to trigger nodulation, which remain to be established. New studies identifying surrogate markers of APA mutational status before surgery, associated with the recent description of drugs specifically blocking mutated KCNJ5 channels, could open new and exciting perspectives for a better diagnosis and targeted treatment 312 of PA.

\section{Acknowledgments}

The laboratory of Dr. Maria-Christina Zennaro is supported through institutional funding from

INSERM, by the Agence Nationale pour la Recherche (ANR-15-CE14-0017-03, ANR-18-

CE93-0003-01), the Fondation pour la Recherche Médicale (EQU201903007864) and by the 


\section{Box 1. Regulation of aldosterone biosynthesis}

322

323

Aldosterone is produced in the zona glomerulosa of the adrenal cortex to maintain blood volume as well as sodium and potassium homeostasis in the body. Aldosterone is synthetized from cholesterol through sequential enzymatic steps catalyzed by different cytochrome P450 enzymes and hydroxysteroid dehydrogenases. In the zona glomerulosa, the enzyme aldosterone synthase (encoded by CYP11B2) catalyzes the three terminal steps of aldosterone biosynthesis: hydroxylation at position $\mathrm{C} 11$ of deoxycorticosterone (DOC) resulting in corticosterone, hydroxylation at position C18 of corticosterone yielding 18-hydroxy-corticosterone and the oxidation of the hydroxyl group at $\mathrm{C} 18$, producing aldosterone.

Aldosterone biosynthesis is mainly regulated by the renin angiotensin system and plasma potassium concentrations. Under basal condition zona glomerulosa cells are hyperpolarized, due to the presence of a large number of $\mathrm{K}^{+}$channels. Both increased extracellular potassium concentrations and angiotensin II inhibit $\mathrm{K}^{+}$channels, leading to cell membrane depolarization, opening of voltage gated $\mathrm{Ca}^{2+}$ channels, increasing intracellular $\mathrm{Ca}^{2+}$ concentrations. Angiotensin II also signals through the Angiotensin II Type 1 receptor via Gaq proteins leading to activation of phospholipase $\mathrm{C}-\beta$, increase of inositol triphosphate, and mobilization of intracellular $\mathrm{Ca}^{2+}$ stores from the endoplasmatic reticulum, increasing intracellular calcium levels. This leads to activation of $\mathrm{Ca}^{2+}$ signaling, which increases aldosterone biosynthesis by activating transcription factors, in particular the nuclear receptor subfamily 4 group A (NR4A) members 1 and 2 (NURR77 or NGF1B and NURR1), the cyclic AMP-dependent transcription factor ATF-1 and the cyclic AMP-responsive element-binding protein (CREB), which increase the expression of $C Y P 11 B 2$, coding for aldosterone synthase. Increased $\mathrm{Ca}^{2+}$ signaling also enhances early and intermediary steps of adrenal steroidogenesis, including release of cholesterol from cytoplasmic stores, activation of the expression of the steroid acute regulatory protein (StAR), which promotes the transfer of cholesterol to the inner mitochondrial membrane, and the synthesis of cofactors for p450 cytochrome enzyme.

\section{Box 2. Clinician's Corner}

Primary aldosteronism (PA) is the most frequent form of secondary arterial hypertension with a prevalence of up to $10 \%$ of hypertensive patients. PA patients have a higher cardiovascular risk compared to patients with essential hypertension. The two main causes of PA are unilateral aldosterone producing adenomas (APA) and bilateral adrenal hyperplasia. The diagnosis of PA includes screening, confirmation tests and subtype identification. Treatment is 
tailored to the underlying cause: adrenalectomy for unilateral disease and drug therapy for bilateral forms.

Familial forms of PA (FH) account for 5\% of the cases. They are classified according to the underlying genetic defect. FH-I is caused by a chimeric gene composed of the promoter regions of $C Y P 11 B 1$ and the coding region from $C Y P 11 B 2$. FH-II is caused by germline mutations in the gene $C L C N 2$, coding for the chloride channel $\mathrm{ClC}-2$. Germline mutations in $K C N J 5$, coding for the potassium channel GIRK4 are responsible FH-III. Finally, germline mutations in $C A C N A 1 H$, coding for the voltage dependent T-type calcium channel Cav 3.2 are responsible by FH-IV. Germline CACNA1D mutations were associated with a rare form of early-onset hyperaldosteronism associated with neurological abnormalities.

Somatic mutations in genes coding for ion channels $(K C N J 5, C A C N A 1 D)$ and ATPases (ATP1A1 and ATP2B3) were identified in the majority of APA. Their frequency may vary in different populations, with KCNJ5 being more frequent in cohorts from Asia and CACNAID mutations in cohorts from African ancestry. KCNJ5 mutations are more frequent in women and young patients. Mutations in $C T N N B 1$, coding for $\beta$-catenin, were identified in $5 \%$ of APA, most frequently in women and possibly associated to pregnancy.

Somatic mutations are also found in aldosterone producing cell clusters (APCC), adrenal structures responsible for autonomous aldosterone production, in normal adrenals. Somatic mutations in APCC are also found in the adrenal cortex adjacent to APA and adrenals from bilateral adrenal hyperplasia. APCC may play a role in the development of PA, as well as in the physiology of aldosterone biosynthesis in normal adrenals.

The discovery of the genetic basis of PA opens new perspectives for the development of new and better diagnostic tools and for drug therapy targeting mutated proteins. Also, it allows for genetic screening and counseling in affected families and improved management of mutation carriers. 


\section{References}

382

383

384

385

386

387

388

389

390

391

392

393

394

395

396

397

398

399

400

401

402

403

404

405

406

407

408

409

410

411

412

1. Monticone, S. et al. (2017) Prevalence and Clinical Manifestations of Primary Aldosteronism Encountered in Primary Care Practice. J Am Coll Cardiol 69 (14), 1811-1820.

2. Hannemann, A. and Wallaschofski, H. (2012) Prevalence of primary aldosteronism in patient's cohorts and in population-based studies--a review of the current literature. Horm Metab Res 44 (3), 157-62.

3. Funder, J.W. et al. (2016) The Management of Primary Aldosteronism: Case Detection, Diagnosis, and Treatment: An Endocrine Society Clinical Practice Guideline. J Clin Endocrinol Metab 101 (5), 1889-916.

4. Savard, S. et al. (2013) Cardiovascular complications associated with primary aldosteronism: a controlled cross-sectional study. Hypertension 62 (2), 331-6.

5. Monticone, S. et al. (2018) Cardiovascular events and target organ damage in primary aldosteronism compared with essential hypertension: a systematic review and meta-analysis. Lancet Diabetes Endocrinol 6 (1), 41-50.

6. Connell, J.M. and Davies, E. (2005) The new biology of aldosterone. J Endocrinol 186 (1), $1-20$.

7. Bandulik, S. et al. (2010) TASK1 and TASK3 potassium channels: determinants of aldosterone secretion and adrenocortical zonation. Horm Metab Res 42 (6), 450-7.

8. Bassett, M.H. et al. (2004) The regulation of aldosterone synthase expression. Mol Cell Endocrinol 217 (1-2), 67-74.

9. Szekeres, M. et al. (2009) Mechanisms of angiotensin II-mediated regulation of aldosterone synthase expression in H295R human adrenocortical and rat adrenal glomerulosa cells. Mol Cell Endocrinol 302 (2), 244-53.

10. Hattangady, N.G. et al. (2012) Acute and chronic regulation of aldosterone production. Mol Cell Endocrinol 350 (2), 151-62.

11. Kim, S.Y. et al. (1998) EGF-stimulated aldosterone secretion is mediated by tyrosine phosphorylation but not by phospholipase $\mathrm{C}$ in cultured porcine adrenal glomerulosa cells. $\mathrm{J}$ Korean Med Sci 13 (6), 629-37.

12. Judd, A.M. et al. (2000) Possible function of IL-6 and TNF as intraadrenal factors in the regulation of adrenal steroid secretion. Ann N Y Acad Sci 917, 628-37.

13. Lefebvre, H. et al. (2019) Paracrine Regulation of Aldosterone Secretion in Physiological and Pathophysiological Conditions. Vitam Horm 109, 303-339. 
14. Lifton, R.P. et al. (1992) A chimaeric 11beta-hydroxylase aldosterone synthase gene causes glucocorticoid-remediable aldosteronism and human hypertension. Nature 355, 262-265. 15. Pascoe, L. et al. (1992) Glucocorticoid-Suppressible Hyperaldosteronism Results from Hybrid Genes Created by Unequal Crossovers Between CYP11B1 and CYP11B2. Proc. Natl. Acad. Sci. - U.S.A. 89, 8327-8331.

16. Sutherland, D.J. et al. (1966) Hypertension, increased aldosterone secretion and low plasma renin activity relieved by dexamethasone. Can Med Assoc J 95 (22), 1109-19.

17. Halperin, F. and Dluhy, R.G. (2011) Glucocorticoid-remediable aldosteronism. Endocrinol Metab Clin North Am 40 (2), 333-41, viii.

18. Pascoe, L. et al. (1995) Glucocorticoid-suppressible hyperaldosteronism and adrenal tumors occurring in a single French pedigree. J Clin Invest 96 (5), 2236-46.

19. Stowasser, M. and Gordon, R.D. (2000) Primary aldosteronism: learning from the study of familial varieties. J Hypertens 18 (9), 1165-76.

20. Choi, M. et al. (2011) K+ channel mutations in adrenal aldosterone-producing adenomas and hereditary hypertension. Science 331 (6018), 768-72.

21. Azizan, E.A. et al. (2013) Somatic mutations in ATP1A1 and CACNA1D underlie a common subtype of adrenal hypertension. Nat Genet 45 (9), 1055-60.

22. Scholl, U.I. et al. (2013) Somatic and germline CACNA1D calcium channel mutations in aldosterone-producing adenomas and primary aldosteronism. Nat Genet 45 (9), 1050-4.

23. Beuschlein, F. et al. (2013) Somatic mutations in ATP1A1 and ATP2B3 lead to aldosteroneproducing adenomas and secondary hypertension. Nat Genet 45 (4), 440-4, 444e1-2.

24. Fernandes-Rosa, F.L. et al. (2014) Genetic spectrum and clinical correlates of somatic mutations in aldosterone-producing adenoma. Hypertension 64 (2), 354-61.

25. Akerstrom, T. et al. (2015) Novel somatic mutations and distinct molecular signature in aldosterone-producing adenomas. Endocr Relat Cancer 22 (5), 735-44.

26. Nanba, K. et al. (2018) Targeted Molecular Characterization of Aldosterone-Producing Adenomas in White Americans. J Clin Endocrinol Metab 103 (10), 3869-3876.

27. Nanba, K. et al. (2019) Genetic Characteristics of Aldosterone-Producing Adenomas in Blacks. Hypertension 73 (4), 885-892.

28. De Sousa, K. et al. (2020) Genetic, Cellular, and Molecular Heterogeneity in Adrenals With Aldosterone-Producing Adenoma. Hypertension, HYPERTENSIONAHA11914177.

29. Scholl, U.I. et al. (2015) Recurrent gain of function mutation in calcium channel CACNA1H causes early-onset hypertension with primary aldosteronism. Elife 4, e06315. 
30. Daniil, G. et al. (2016) CACNA1H Mutations Are Associated With Different Forms of Primary Aldosteronism. EBioMedicine 13, 225-236.

31. Fernandes-Rosa, F.L. et al. (2018) A gain-of-function mutation in the CLCN2 chloride channel gene causes primary aldosteronism. Nat Genet 50 (3), 355-361.

32. Scholl, U.I. et al. (2018) CLCN2 chloride channel mutations in familial hyperaldosteronism type II. Nat Genet 50 (3), 349-354.

33. Oki, K. et al. (2012) The potassium channel, Kir3.4 participates in angiotensin II-stimulated aldosterone production by a human adrenocortical cell line. Endocrinology 153 (9), 4328-35.

34. Murthy, M. et al. (2012) Characterization of a novel somatic KCNJ5 mutation delI157 in an aldosterone-producing adenoma. J Hypertens 30 (9), 1827-33.

35. Cheng, C.J. et al. (2015) Novel KCNJ5 mutations in sporadic aldosterone-producing adenoma reduce Kir3.4 membrane abundance. J Clin Endocrinol Metab 100 (1), E155-63. 36. Yang, Y. et al. (2019) Primary Aldosteronism: KCNJ5 Mutations and Adrenocortical Cell Growth. Hypertension 74 (4), 809-816.

37. Azizan, E.A. et al. (2012) Somatic mutations affecting the selectivity filter of KCNJ5 are frequent in 2 large unselected collections of adrenal aldosteronomas. Hypertension 59 (3), 58791.

38. Lenzini, L. et al. (2015) A Meta-Analysis of Somatic KCNJ5 K(+) Channel Mutations In 1636 Patients With an Aldosterone-Producing Adenoma. J Clin Endocrinol Metab 100 (8), E1089-95.

39. Taguchi, R. et al. (2012) Expression and mutations of KCNJ5 mRNA in Japanese patients with aldosterone-producing adenomas. J Clin Endocrinol Metab 97 (4), 1311-9.

40. Zheng, F.F. et al. (2015) Clinical characteristics of somatic mutations in Chinese patients with aldosterone-producing adenoma. Hypertension 65 (3), 622-8.

41. Wang, B. et al. (2015) Prevalence and characterization of somatic mutations in Chinese aldosterone-producing adenoma patients. Medicine (Baltimore) 94 (16), e708.

42. Boulkroun, S. et al. (2012) Prevalence, clinical, and molecular correlates of KCNJ5 mutations in primary aldosteronism. Hypertension 59 (3), 592-8.

43. Scholl, U.I. et al. (2015) Novel somatic mutations in primary hyperaldosteronism are related to the clinical, radiological and pathological phenotype. Clin Endocrinol (Oxf) 83 (6), 779-89. 44. Vilela, L.A.P. et al. (2019) KCNJ5 somatic mutation is a predictor of hypertension remission after adrenalectomy for unilateral primary aldosteronism. J Clin Endocrinol Metab 104 (10), 4695-4702. 
45. Yamazaki, Y. et al. (2017) Histopathological Classification of Cross-Sectional ImageNegative Hyperaldosteronism. J Clin Endocrinol Metab 102 (4), 1182-1192.

46. Boulkroun, S. et al. (2013) KCNJ5 mutations in aldosterone producing adenoma and relationship with adrenal cortex remodeling. Mol Cell Endocrinol 371 (1-2), 221-7.

47. Fernandes-Rosa, F.L. et al. (2015) Functional histopathological markers of aldosterone producing adenoma and somatic KCNJ5 mutations. Mol Cell Endocrinol 408, 220-6.

48. Geller, D.S. et al. (2008) A novel form of human mendelian hypertension featuring nonglucocorticoid-remediable aldosteronism. J Clin Endocrinol Metab 93 (8), 3117-23.

49. Mulatero, P. et al. (2013) Role of KCNJ5 in familial and sporadic primary aldosteronism. Nat Rev Endocrinol 9 (2), 104-12.

50. Scholl, U.I. et al. (2012) Hypertension with or without adrenal hyperplasia due to different inherited mutations in the potassium channel KCNJ5. Proc Natl Acad Sci U S A 109 (7), 25338.

51. Mulatero, P. et al. (2012) KCNJ5 mutations in European families with nonglucocorticoid remediable familial hyperaldosteronism. Hypertension 59 (2), 235-40.

52. Monticone, S. et al. (2013) a Novel Y152C KCNJ5 mutation responsible for familial hyperaldosteronism type III. J Clin Endocrinol Metab 98 (11), E1861-5.

53. Adachi, M. et al. (2014) Discordant Genotype-Phenotype Correlation in Familial Hyperaldosteronism Type III with KCNJ5 Gene Mutation: A Patient Report and Review of the Literature. Horm Res Paediatr 82 (2), 138-42.

54. Tauber, P. et al. (2014) Pharmacology and pathophysiology of mutated KCNJ5 found in adrenal aldosterone producing adenomas. Endocrinology, en20131944.

55. Scholl, U.I. et al. (2017) Macrolides selectively inhibit mutant KCNJ5 potassium channels that cause aldosterone-producing adenoma. J Clin Invest 127 (7), 2739-2750.

56. Caroccia, B. et al. (2017) Macrolides Blunt Aldosterone Biosynthesis: A Proof-of-Concept Study in KCNJ5 Mutated Adenoma Cells Ex Vivo. Hypertension 70 (6), 1238-1242.

57. Maiolino, G. et al. (2018) Macrolides for KCNJ5-mutated aldosterone-producing adenoma (MAPA): design of a study for personalized diagnosis of primary aldosteronism. Blood Press 27 (4), 200-205.

58. Nanba, K. et al. (2020) Somatic CACNA1H Mutation As a Cause of Aldosterone-Producing Adenoma. Hypertension 75 (3), 645-649.

59. Williams, T.A. et al. (2014) Somatic ATP1A1, ATP2B3, and KCNJ5 mutations in aldosterone-producing adenomas. Hypertension 63 (1), 188-95. 
60. Stindl, J. et al. (2015) Pathogenesis of Adrenal Aldosterone-Producing Adenomas Carrying Mutations of the $\mathrm{Na}(+) / \mathrm{K}(+)-A T P a s e$. Endocrinology 156 (12), 4582-91.

61. Li, C. et al. (2005) A third Na+-binding site in the sodium pump. Proc Natl Acad Sci U S A 102 (36), 12706-11.

62. Dutta, R.K. et al. (2014) Complementary somatic mutations of KCNJ5, ATP1A1, and ATP2B3 in sporadic aldosterone producing adrenal adenomas. Endocr Relat Cancer 21 (1), L14.

63. Tauber, P. et al. (2016) Cellular Pathophysiology of an Adrenal Adenoma-Associated Mutant of the Plasma Membrane Ca(2+)-ATPase ATP2B3. Endocrinology 157 (6), 2489-99.

64. Stowasser, M. et al. (1992) Familial hyperaldosteronism type II: five families with a new variety of primary aldosteronism. Clin Exp Pharmacol Physiol 19 (5), 319-22.

65. Stowasser, M. and Gordon, R.D. (2001) Familial hyperaldosteronism. J Steroid Biochem Mol Biol 78 (3), 215-29.

66. Dutta, R.K. et al. (2019) A somatic mutation in CLCN2 identified in a sporadic aldosteroneproducing adenoma. Eur J Endocrinol 181 (5), K37-K41.

67. Akerstrom, T. et al. (2016) Activating mutations in CTNNB1 in aldosterone producing adenomas. Sci Rep 6, 19546.

68. Teo, A.E. et al. (2015) Pregnancy, Primary Aldosteronism, and Adrenal CTNNB1 Mutations. N Engl J Med 373 (15), 1429-36.

69. Gagnon, N. et al. (2018) Genetic Characterization of GnRH/LH-Responsive Primary Aldosteronism. J Clin Endocrinol Metab 103 (8), 2926-2935.

70. Rhayem, Y. et al. (2016) PRKACA Somatic Mutations Are Rare Findings in AldosteroneProducing Adenomas. J Clin Endocrinol Metab 101 (8), 3010-7.

71. Zilbermint, M. et al. (2015) Primary Aldosteronism and ARMC5 Variants. J Clin Endocrinol Metab 100 (6), E900-9.

72. Mulatero, P. et al. (2016) ARMC5 mutation analysis in patients with primary aldosteronism and bilateral adrenal lesions. J Hum Hypertens 30 (6), 374-8.

73. Assie, G. et al. (2013) ARMC5 mutations in macronodular adrenal hyperplasia with Cushing's syndrome. N Engl J Med 369 (22), 2105-14.

74. Heitzmann, D. et al. (2008) Invalidation of TASK1 potassium channels disrupts adrenal gland zonation and mineralocorticoid homeostasis. EMBO J 27 (1), 179-87.

75. Davies, L.A. et al. (2008) TASK channel deletion in mice causes primary hyperaldosteronism. Proc Natl Acad Sci U S A 105 (6), 2203-8. 
76. Penton, D. et al. (2012) Task3 potassium channel gene invalidation causes low renin and salt-sensitive arterial hypertension. Endocrinology 153 (10), 4740-8.

77. Guagliardo, N.A. et al. (2012) TASK-3 channel deletion in mice recapitulates low-renin essential hypertension. Hypertension 59 (5), 999-1005.

78. Guagliardo, N.A. et al. (2019) Adrenal Tissue-Specific Deletion of TASK Channels Causes Aldosterone-Driven Angiotensin II-Independent Hypertension. Hypertension 73 (2), 407-414. 79. Bandulik, S. et al. (2013) Severe hyperaldosteronism in neonatal Task3 potassium channel knockout mice is associated with activation of the intraadrenal renin-angiotensin system. Endocrinology 154 (8), 2712-22.

80. El Wakil, A. et al. (2012) Dkk3 is a component of the genetic circuitry regulating aldosterone biosynthesis in the adrenal cortex. Hum Mol Genet 21 (22), 4922-9.

81. Goppner, C. et al. (2019) Pathogenesis of hypertension in a mouse model for human CLCN2 related hyperaldosteronism. Nat Commun 10 (1), 4678.

82. Schewe, J. et al. (2019) Elevated aldosterone and blood pressure in a mouse model of familial hyperaldosteronism with ClC-2 mutation. Nat Commun 10 (1), 5155.

83. Billet, S. et al. (2007) Gain-of-function mutant of angiotensin II receptor, type 1A, causes hypertension and cardiovascular fibrosis in mice. J Clin Invest 117 (7), 1914-25.

84. Taylor, M.J. et al. (2020) Chemogenetic activation of adrenocortical Gq signaling causes hyperaldosteronism and disrupts functional zonation. J Clin Invest 130 (1), 83-93.

85. Tissier, F. et al. (2005) Mutations of beta-catenin in adrenocortical tumors: activation of the Wnt signaling pathway is a frequent event in both benign and malignant adrenocortical tumors. Cancer Res 65 (17), 7622-7.

86. Boulkroun, S. et al. (2011) Aldosterone-producing adenoma formation in the adrenal cortex involves expression of stem/progenitor cell markers. Endocrinology 152 (12), 4753-63.

87. Berthon, A. et al. (2014) WNT/beta-catenin signalling is activated in aldosterone-producing adenomas and controls aldosterone production. Hum Mol Genet 23 (4), 889-905.

88. Dihazi, G.H. et al. (2015) Proteomic characterization of adrenal gland embryonic development reveals early initiation of steroid metabolism and reduction of the retinoic acid pathway. Proteome Sci 13, 6.

89. El Zein, R.M. et al. (2019) Retinoic acid receptor alpha as a novel contributor to adrenal cortex structure and function through interactions with Wnt and Vegfa signalling. Sci Rep 9 (1), 14677.

90. Nishimoto, K. et al. (2010) Adrenocortical zonation in humans under normal and pathological conditions. J Clin Endocrinol Metab 95 (5), 2296-305. 
579 91. Boulkroun, S. et al. (2010) Adrenal cortex remodeling and functional zona glomerulosa 580 hyperplasia in primary aldosteronism. Hypertension 56 (5), 885-92.

581 92. Nishimoto, K. et al. (2015) Aldosterone-stimulating somatic gene mutations are common 582 in normal adrenal glands. Proc Natl Acad Sci U S A 112 (33), E4591-9.

583 93. Nishimoto, K. et al. (2017) Immunohistochemistry of aldosterone synthase leads the way 584 to the pathogenesis of primary aldosteronism. Mol Cell Endocrinol 441, 124-133.

585 94. Omata, K. et al. (2018) Cellular and Genetic Causes of Idiopathic Hyperaldosteronism. 586 Hypertension 72 (4), 874-880.

587 95. Gomez-Sanchez, C.E. and Gomez-Sanchez, E.P. (2012) Mutations of the potassium channel 588 KCNJ5 causing aldosterone-producing adenomas: one or two hits? Hypertension 59 (2), 1965897.

590 96. Fernandes-Rosa, F.L. et al. (2015) Different Somatic Mutations in Multinodular Adrenals 591 With Aldosterone-Producing Adenoma. Hypertension 66 (5), 1014-22.

592 97. Vouillarmet, J. et al. (2016) Aldosterone-Producing Adenoma With a Somatic KCNJ5 593 Mutation Revealing APC-Dependent Familial Adenomatous Polyposis. J Clin Endocrinol 594 Metab 101 (11), 3874-3878.

595 


\section{Figure legends}

Figure 1. Cellular mechanisms of autonomous aldosterone biosynthesis. In unstimulated conditions, zona glomerulosa cells are in a hyperpolarized state, due to the presence of a large number of potassium channels. Cell membrane depolarization, following stimulation of aldosterone biosynthesis, leads to opening of voltage-gated calcium channels and activation of calcium signaling, the main trigger for aldosterone biosynthesis. Mutations in different channels and pumps have been identified in different forms of familial hyperaldosteronism and APA. Mutations in GIRK4 potassium channels in APA and FH-III lead to sodium entry into the cells instead of extrusion of potassium. Mutations in CLC-2 chloride channels in FH-II and APA increase chloride efflux, and mutations in the $\alpha 1$ subunit of the $\mathrm{Na}^{+}, \mathrm{K}^{+}$-ATPase, found in APA, are responsible for loss of pump activity, but also lead to proton entry into the cells. These mutations lead to cell membrane depolarization and opening of voltage gated calcium channels. Mutations in the $\alpha 1$ subunit of the L-type $\mathrm{Ca}^{2+}$ channel Cav1.3, found in APA and PASNA, and in $\alpha 1$ subunit of the T-type $\mathrm{Ca}^{2+}$ channel Cav3.2 in FH-IV and APA, result in changes in their functional properties, particularly allowing calcium entry at lower membrane potential, whereas mutations in PMCA3 are responsible for loss of pump activity and accumulation of calcium in the cells. All these mutations induce an increase of intracellular calcium concentration and activation of calcium signaling, followed by stimulation of CYP11B2 transcription and increase in aldosterone biosynthesis. In FH-I, a chimeric gene in which the coding region of CYP11B2 is fused to the regulatory region of $C Y P 11 B 1$ leads to ectopic expression of $C Y P 11 B 2$ throughout the adrenal cortex and control of aldosterone biosynthesis mainly by ACTH.

Figure 2: Pathogenic model of aldosterone producing adenoma development. Two different models may explain APA development in the adrenal cortex. (Left panel) "APCC model": occurrence of somatic mutations in different APA driver genes (KCNJ5, CACNA1D, $A T P 1 A 1, A T P 2 B 3, K C N J 5)$ lead to the development of aldosterone producing cell clusters (APCC). APCC may develop into APA through the formation of possible APCC-to-APA translational lesions (pAATL). (Right panel) "Two-hit model”: Abnormal cell proliferation and nodulation in the zona glomerulosa, due to genetic or environmental factors, may create a propitious environment for the occurrence of somatic mutations in APA driver genes (KCNJ5, CACNA1D, ATP1A1, ATP2B3, CACNA1H, CLCN2). In both hypothesis, activation of calcium signaling, due to the presence of somatic mutations, leads to increased CYP11B2 expression and autonomous aldosterone production. 
Table 1. Familial and inherited forms of primary aldosteronism.

\begin{tabular}{|c|c|c|c|c|c|}
\hline Disease & Age of onset & Specific features & Gene & Transmission & Treatment \\
\hline $\begin{array}{l}\text { Familial hyperaldosteronism } \\
\text { type I }\end{array}$ & $\begin{array}{l}\text { Variable } \\
\text { Often before } 20 \text { ys }\end{array}$ & $\begin{array}{l}\text { Cerebrovascular } \\
\text { events at young age } \\
(<30 \text { ys })\end{array}$ & $\begin{array}{l}\text { Chimeric } \\
C Y P 11 B 1 / B 2\end{array}$ & $\mathrm{AD}$ & Glucocorticoids, MRA \\
\hline $\begin{array}{l}\text { Familial hyperaldosteronism } \\
\text { type II }\end{array}$ & $\begin{array}{l}\text { Variable } \\
\text { Young onset in } \\
\text { patients with } \\
\text { CLCN2 mutations }\end{array}$ & none & CLCN2 & $\mathrm{AD}$ & MRA \\
\hline $\begin{array}{l}\text { Familial hyperaldosteronism } \\
\text { type III }\end{array}$ & $\begin{array}{l}\text { Before } 20 \text { ys } \\
\text { Variable in mild } \\
\text { forms }\end{array}$ & $\begin{array}{l}\text { Massive bilateral } \\
\text { adrenal hyperplasia } \\
\text { in severe cases }\end{array}$ & KCNJ5 & $\mathrm{AD}$ & $\begin{array}{l}\text { MRA } \\
\text { Bilateral adrenalectomy in } \\
\text { severe cases }\end{array}$ \\
\hline $\begin{array}{l}\text { Familial hyperaldosteronism } \\
\text { type IV }\end{array}$ & $\begin{array}{l}\text { Variable } \\
\text { Most frequent } \\
\text { before } 20 \text { ys }\end{array}$ & $\begin{array}{l}\text { Developmental } \\
\text { disorder in some } \\
\text { cases }\end{array}$ & CACNAIH & $\mathrm{AD}$ & MRA \\
\hline $\begin{array}{l}\text { Primary Aldosteronism, } \\
\text { Seizures and Neurologic } \\
\text { Abnormalities (PASNA) }\end{array}$ & Childhood & $\begin{array}{l}\text { Seizures and } \\
\text { neurological } \\
\text { abnormalities }\end{array}$ & CACNAID & ? (de novo) & Calcium channel blocker \\
\hline
\end{tabular}


Table 2. Recent mouse models of hyperaldosteronism.

\begin{tabular}{|c|c|c|c|c|}
\hline Model & Phenotype & Adrenal Phenotype & Mechanism & Ref \\
\hline TASK1 inactivation & $\begin{array}{l}\text { - Hyperaldosteronism remediable } \\
\text { by glucocorticoids } \\
\text { - } \quad \text { Both sexes in young mice } \\
\text { - } \text { Restricted to females in adult mice }\end{array}$ & $\begin{array}{l}\text { - } \text { Cyp11B2 absent in the } \mathrm{ZG} \\
\text { - Ectopic Cyp11B2 expression in } \\
\text { the ZF restricted to females in } \\
\text { adults. }\end{array}$ & $\begin{array}{l}\text { More depolarized membrane } \\
\text { voltage at resting conditions }\end{array}$ & [74] \\
\hline $\begin{array}{l}\text { TASK1 and TASK3 } \\
\text { inactivation }\end{array}$ & $\begin{array}{l}\text { - Overproduction of aldosterone } \\
\text { resistant to salt suppression } \\
\text { - Normal/low renin }\end{array}$ & $\begin{array}{l}\text { - Cellular morphology and zonation } \\
\text { preserved in adult mice (only } \\
\text { males) }\end{array}$ & $\begin{array}{l}\text { - Marked depolarization of ZG } \\
\text { cells. }\end{array}$ & [75] \\
\hline TASK3 inactivation & $\begin{array}{l}\text { - Salt-sensitive, low renin, arterial } \\
\text { hypertension } \\
\text { - Abnormalities in the regulation of } \\
\text { aldosterone } \\
\text { - Severe hyperaldosteronism in } \\
\text { neonatal knockout mice }\end{array}$ & $\begin{array}{l}\text { - No changes in adrenal } \\
\text { morphology }\end{array}$ & $\begin{array}{l}\text { Depolarization of } \\
\text { adrenocortical cells. } \\
\text { - Abnormal } \mathrm{Ca}^{2+} \text { signaling. }\end{array}$ & {$[76,79]$} \\
\hline TASK3 inactivation & $\begin{array}{l}\text { - Salt-sensitive, low renin, arterial } \\
\text { hypertension } \\
\text { - Mild hyperaldosteronism resistant } \\
\text { to salt suppression }\end{array}$ & $\begin{array}{l}\text { - No changes in adrenal } \\
\text { morphology }\end{array}$ & $\begin{array}{l}\text { More depolarized membrane } \\
\text { voltage at resting conditions }\end{array}$ & [77] \\
\hline $\begin{array}{l}\text { TASK1 and DKK3 } \\
\text { inactivation }\end{array}$ & $\begin{array}{l}\text { Hyperaldosteronism remediable } \\
\text { by glucocorticoids in both sexes }\end{array}$ & $\begin{array}{l}\text { - Absence of altered functional } \\
\text { zonation in male mice }\end{array}$ & $\begin{array}{l}\text { More depolarized membrane } \\
\text { voltage at resting conditions }\end{array}$ & {$[80]$} \\
\hline $\begin{array}{l}\text { Constitutive activation } \\
\text { of } \beta \text {-catenin }\end{array}$ & $\begin{array}{l}\text { Increased aldosterone production } \\
\text { independent of renin }\end{array}$ & $\begin{array}{l}\text { Ectopic expression of Dab2, Beta- } \\
\text { catenin and Cyp11b2 in ZF }\end{array}$ & $\begin{array}{l}\text { - Beta-catenin dependent } \\
\text { activation of at1r and cyp21 } \\
\text { - Increased expression of } \\
\text { Nurr } 1 \text { and Nur77 leading to } \\
\text { Cyp } 11 b 2 \text { activation }\end{array}$ & [87] \\
\hline $\begin{array}{l}\text { ZG Specific } \\
\text { inactivation of TASK1 } \\
\text { and TASK } 3\end{array}$ & $\begin{array}{ll}\text { - } & \text { Arterial hypertension } \\
\text { - } & \text { Mild hyperaldosteronism } \\
\text { - } & \text { Mild aldosterone autonomy }\end{array}$ & NA & NA & [78] \\
\hline
\end{tabular}


Constitutively open

ClC-2

Marked hypertension

- High serum aldosterone

- Low renin activity

- Hypokalemia

- Mildly elevated blood pressure

- Mildly elevated aldosterone levels

nnock-in Clcn2 model (homologous to human CLCN2 mutation in FH-II)

Specific and inducible adrenal activation of

Gq signaling

- Hypertension

- Increased aldosterone levels

- Decreased renin
- No changes in adrenal morphology, increased expression of Cyp11b2 in the ZG

- Increased chloride conductance in the ZG

- Cell membrane depolarization

- Increased cytoplasmic $\mathrm{Ca}^{2+}$ concentration

- Increased Cyp11b2 expression

- No changes in adrenal morphology

- Incomplete suppression of Cyp11b2 expression upon highsalt challenge

- Disorganization of adrenal functional zonation

- Increased calcium oscillatory activity in adrenal cells

- Increased Cyp11b2 expression

- Gq signaling dependent Cyp1 1b2 expression in ZF cells

ZG: zona glomerulosa; ZF: zona fasciculata; NA: not available 


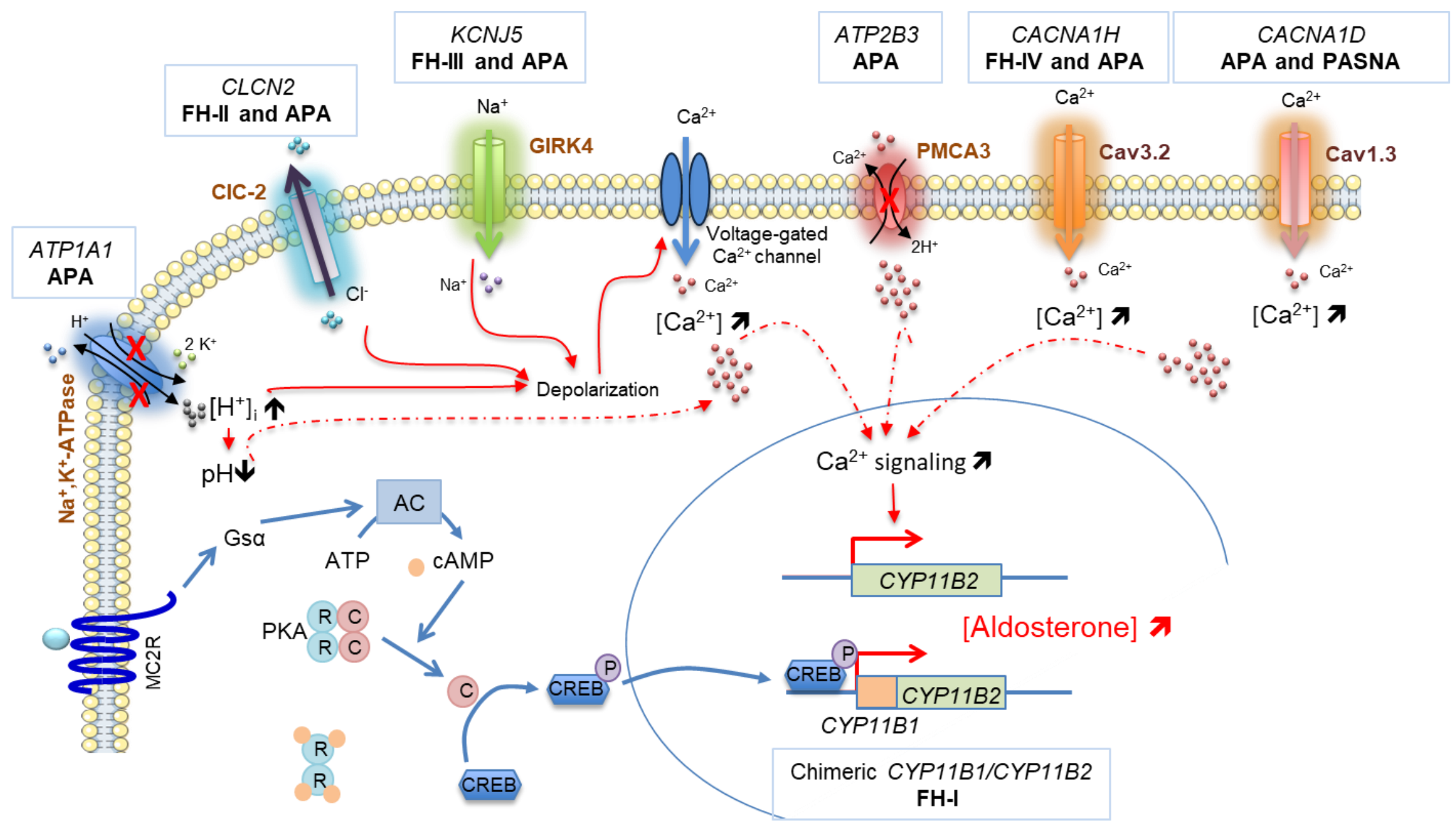

Figure 1 


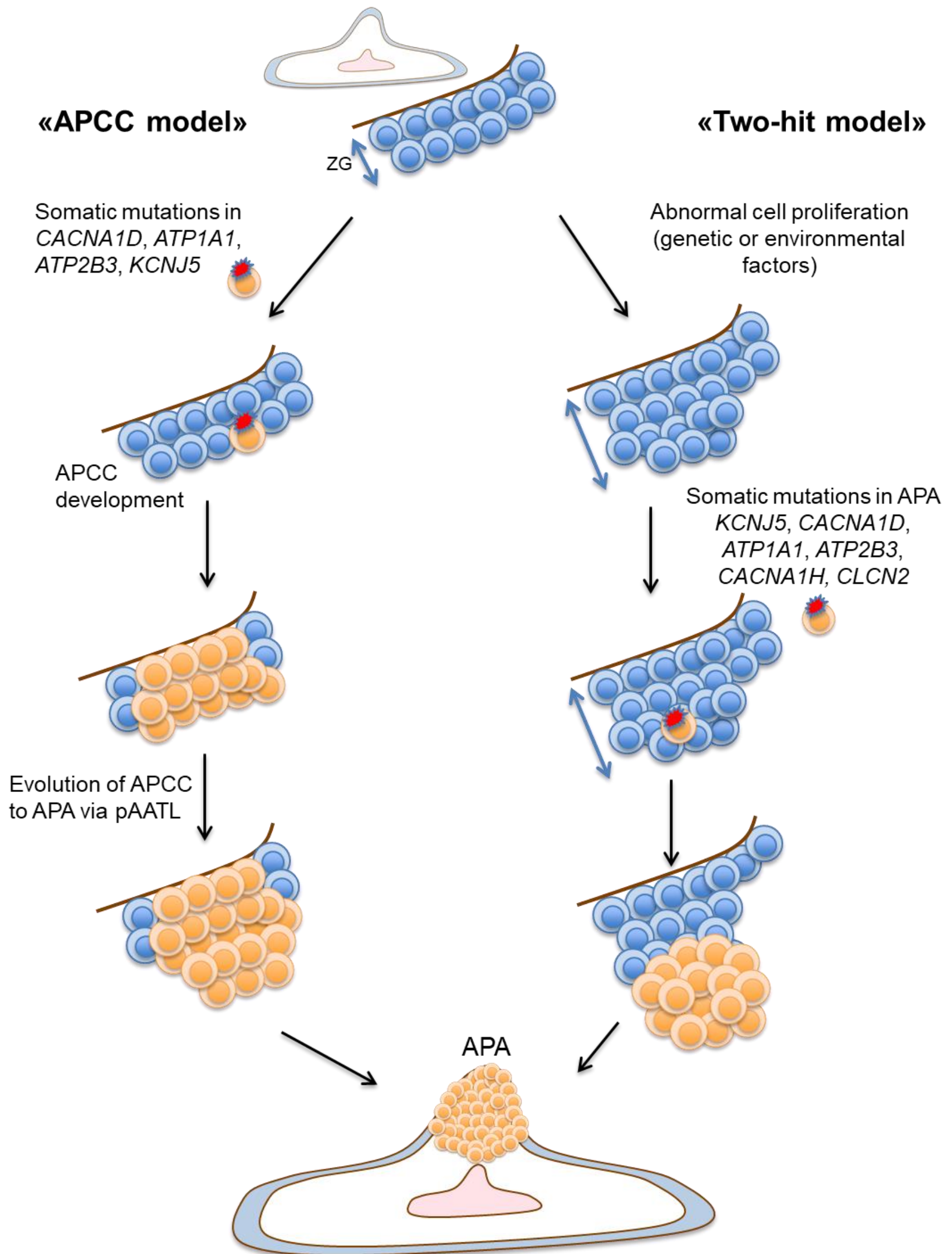

Figure 2 
